\title{
Clinical teaching fellowships - enhancing the out of programme experience through a peer network
}

Editor - The Royal College of Physicians is turning its focus towards sustainable working practice in the UK. ${ }^{1}$ This includes job plans which provide time for education, research or innovation in addition to clinical work. Clinical teaching fellowships offer an opportunity for junior doctors to dedicate time to teaching without the competing interests of service provision. ${ }^{2}$ They are usually 1-year posts, which can involve bedside teaching, assessments, simulation and curriculum development. Job descriptions are diverse, with potential for involvement in pastoral care, educational research and postgraduate educational qualifications. Clinical teaching fellows (CTFs) can be appointed by medical schools, NHS trusts, medical royal colleges and postgraduate deaneries. The clinical commitment varies - many CTFs are not expected to have any clinical role, some negotiate clinical sessions, others may be on a regular rota.

Positions are taken up at various stages in training, either during a natural break (eg between core/specialty training) or as an out of programme experience (OOPE). There are also some career posts with potential for extended contracts.

At our medical school (Imperial College London), CTF numbers are increasing: there have been approximately 190 CTFs in the past 5 years (2014-19). The majority of CTFs in our institution are employed by NHS trusts and have honorary contracts with the university. At other organisations, CTFs are appointed centrally by the university.

The need to support CTFs and encourage professional development during their OOPE was recognised at our institution by senior CTFs and faculty. This was addressed by forming a CTF network in 2014 and was initially supported through an electronic mailing list connecting CTFs from over 10 different NHS sites. This developed into monthly educational meetings and workshops, plus a regular newsletter. A centralised induction and handover booklet to orientate new CTFs was established. Most recently, educational workshops by Medical Education Research Unit offer CTFs a tailored educational programme to gain accreditation with the Academy of Medical Educators.

CTFs have identified valuable aspects of the network to include access to peer support, learning from educational experts and career guidance. The success and sustainability of the network in our region has been due to several factors: firstly, a network chair has been essential to coordinating the activities of the network each year - succession planning was important and so the medical school agreed for this role to be incorporated into the job description of a senior CTF; secondly, outgoing CTFs generated institutional memory by creating handover resources and finally, the CTF network has maintained a good relationship with the medical school faculty and Imperial's Educational Development Unit and Medical Education Research Unit.

At our institution, CTFs play an integral role, valued by students and faculty. The peer network provides a supportive and tailored educational community that encourages development of advanced teaching skills, academic projects and leadership opportunities. By enhancing their OOPEs and developing their portfolios, we hope that the network can benefit the professional development of junior doctors as clinicians, educators and healthcare leaders of the future.

ANN CHU

Academic clinical fellow renal medicine/medical education, Imperial College London, London, UK

CAROLINE MORTON

Educational research fellow, Medical Education Research Unit, Imperial College London, London, UK

CATHERINE PYE

Clinical teaching fellow, Imperial College London, London, UK

LUBNA GHANI

Clinical teaching fellow, Imperial College London, London, UK

SUSAN F SMITH

Professor of medical education, director of Medical Education Research Unit, Imperial College London, London, UK

\section{References}

1 Nicol E. Optimising clinical practice to support a sustainable NHS. Clin Med 2018;18:193.

2 Anyiam $\mathrm{O}$, Mercer C, Zaheen $\mathrm{H}$. Participation in teaching opportunities during core medical training: barriers and enablers. Future Hosp J 2017:4;86-91.

Address for correspondence: $\mathrm{Dr}$ Ann Chu, academic clinical fellow renal medicine/medical education, Faculty of Medicine - North West London Hospitals Campus, Faculty Education Office, Reynolds Building, St Dunstan's Road, Imperial College London, London SW7 2AZ, UK.

Email:ann.chu@imperial.ac.uk 